Aus dem Kantonsspital Winterthur (Direktor: Dr. R. Stierlin) und dem Patholog. Institut der Universität Zürich (Direktor: Prof. Dr. O. Bus s e).

\title{
Über ein angeblich nach Trauma entstandenes, primäres Sarkom der Leber.
}

Von Dr. J. Dubs, Sekundararzt der chirurgischen Abteilung am Kantonsspital Winterthur.

(Mit 3 Abbildungen.)

Klinisch und pathologisch-anatomisch einwandfrei festgestellte, primäre Lebersarkome sind sehr selten und in der Literatur nur in vereinzelten Beobachtungen niedergelegt. Aus der gesamten Literatur konnte Arnold I89o bloß 23 Fälle zusammenstellen. S ch ei d e man d el erwähnt in seiner Arbeit $190327 \mathrm{Be}-$ obachtungen von primärem Lebersarkom, wovon er aber nur i8 als sicher primär gelten lassen will. Marx zählt I9O4 50 Fälle, wovon er 19 als nicht sicher festgestellt betrachtet. Neuerdings hat Thöle I9I3 in seiner Monographie der Lebergeschwülste weitere I4 Publikationen zusammengestellt; in der mir zugänglichen Literatur habe ich seither nur ganz wenige Beobachtungen erwähnt gefunden (Rolleston und Salisbury, Wendel, Picone).

Von 94 durch Garbarini (zit. nach Thöle) zusammengestellten Fällen waren die meisten metastatische; in andern handelte es sich um Tuberkulose, Lues, Leukämie. Das gleiche gilt nach Thöle für die Zusammenstellung von $\mathrm{Kn}$ ot t.

Die Veröffentlichung eines im Kantonsspital Winterthur klinisch und pathologisch-anatomisch genau beobachteten Falles von primärem Lebersarkom erscheint nicht nur als kasuistische Mitteilung berechtigt; sie ist es auch deswegen, weil sie einen nicht uninteressanten Beitrag zur Frage der $\mathrm{tra}$ u mat is chen Ent. s t e h u $\mathrm{n} g$ des Sarkoms darstellt. Gerade in dieser Hinsicht haben 
Autoritäten wie $\mathrm{Th}$ ie $\mathrm{m}$ und $\mathrm{Kaufmann}$ die Wichtigkeit klinisch und pathologischanatomisch gut fundierter $\mathrm{E}$ inzelbeobachtungen besonders betont. - Die Worte Tillmanns

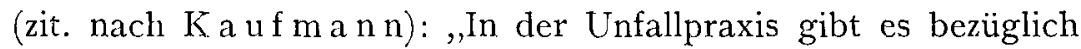
der traumatischen Entstehung der Geschwülste keine :llgemeine Regel; man wird jeden einzelnen Fall für sich betrachten müssen“, werden auch für unseren Fall bestimmend sein, dessen Krankengeschichte hier nun zunächst folgen soll. -

J.-Nr. 15/162I. H. B., 25 Jahre alt, Dienstmädchen in W. gelangt am 7.X. I9I5 auf der chirurgischen Abteilung zur Aufnahme mit folgender An a mnese:

Pat. gibt an, bisher nie ernstlich krank und immer ganz gesund gewesen zu sein. Die Eltern seien an ihr unbekannter Krankheit gestorben, ebenso eine Schwester. Ein Bruder der Pat. lebt und ist gesund. - Im rechten Auge habe sie vor ca. I3 Jahren eine langandauernde „Entzündung" durchgemacht, es sei dann in der Hornhaut nahe der Pupille der jetzt noch bestehende große, weiße Fleck zurückgeblieben. - Irgendwelche erblichen Krankheiten kommen in der Familie oder Verwandtschaft nicht vor; Pat. erinnert sich auch nicht, daß darin Geschwülste auffallend häufig seien.

Pat. ist seit längerer Zeit als Dienstmädchen in W. in Stellung. Sie gibt ausdrücklich an, daß sie immer ganz gesund und munter gewesen sei und ihre Arbeit anstandslos und ohne Beschwerden besorgt habe bis zum 5. IX. Igr 5 .

Menses regelmäßig, alle 4 Wochen, ohne Bcschwerden. Schlaf und Appetit gut, Defäkation und Exurese ohne Besonderheiten.

Pat. gibt auch ausdrücklich an, daß sie bisher weder in der rechten Brust- noch in der Bauchgegend irgendwelche Schmerzen verspürt habe.

Sic sei nie bisher wegen einer Geschwulst operiert worden.

Am 5. IX. I 9 I 5 hatte sie Teppiche zu reinigen und jagte sich dabei mit dem 7 jährigen Söhnchen ihrer Herrschaft herum. Dabei habe - in Gegenwart eines andern Dienstmädchens - der Knabe ihr aus einer Entfernung von ca. 3-4 $\mathrm{m}$ eine schwere, harthölzerne Bürste (Teppichbürste) mit großer Gewalt in die Gegend des rechten Rippenkorbrandes angeworfen. Der Knabe wird von der Pat. als für sein Alter außerordentlich stark und kräftig geschildert. -

Der Schlag durch diesen Bürstenwurf sei so heftig gewesen, daß die Pat. taumelte. Sie verspürte sofort sehr starke Schmerzen in der Gegend des rechten Rippenkorbrandes; es wurde ihr ganz schwindelig; so daß sie sich setzen mußte. Sie habe eine Zeitlang fast nicht mehr atmen können und sei - auch nach der Aussage des andern Dienstmädchens - totenbleich gewesen. 
Nach und nach erholte sie sich etwas; sie versprach auch dem geängstigten Knaben, den Eltern nichts zu sagen. Von diesem Moment an habe sie aber fortwährend starke Schmerzen in der Lebergegend verspürt, besonders bei der Hausarbeit, die sie bis zu dem Unfalle immer leicht und anstandslos besorgt habe. Die Schmerzen seien bald so bedeutend geworden, daß sie ca. 4 Tage nach dem Unfall sich der Herrschaft krank meldete und von ihr sofort in ärztliche Untersuchung und Behandlung gewiesen wurde.

Laut ärztlichem Zeugnis und freundl. Auskunft des behandelnden Arztes wurde damals, also vier Tage nach dem Unfall, folgender Befund erhoben: Die Gegend des rechten Oberbauches sowohl wie dic rechte Thoraxseite, auf die nach Aussage der Pat. der Unfall ein. gewirkt hat, zeigen bei der Inspektion nichts Auffallendes. - Vor allem sind keinerlei objektive Quetschungssymptome, keine Hautverfärbungen zu konstatieren. Auch bei der Palpation habe sich ein abnormer Befund nicht feststellen lassen; sämtliche in Frage kommenden Rippen seien intakt, die Lebergegend bei der Palpation auf Druck leicht schmerzhaft, aber sonst ohne Veränderungen gewesen. - Pat. wurde dann 2 Wochen lang wegen „Quetschung der rechten Rippenbogengegend" ärztlich behandelt und lag dabei zu Bett. - Irgendeine Tumorbildung in der betr. Gegend habe der behandelnde Arzt nicht konstatiert. Nach diesen zwei Wochen wieder für arbeitsfähig erklärt,' arbeitete die Pat. wieder zwei Wochen. Sie habe während dieser Zeit immer Schmerzen in der Lebergegend verspürt, keinen Appetit und keinen guten Schlaf gehabt und immer schlechter ausgesehen. Sie konsultierte deshalb Anfang Oktober also ca. 4 Wochen nach dem Unfall - Herrn Direktor Dr. Stie r lin, der sie auf seinen Befund hin veranlaßte, auf die chirurgische Abteilung des Kantonsspitals Winterthur sich aufnehmen zu lassen. -

$\mathrm{Nach}$ träglich e Erhebungen ergaben nun folgende interessante Ergänzungen zu der Beschreibung des Unfalls und der Erzählung des bisherigen Krankeitsverlautes von seiten der Pat.:

Nach Aussage des zweiten, beim Unfall zugegen gewesenen Dienstmädchens und des 7 jährigen Sohnes der Dienstherrschaft spielte sich der Unfall im wesentlichen so ab, wie geschildert.

Der Knabe, der von der Patientin ,als für sein Alter außerordentlich kräftig und stark" geschildert wurde, ist - wie ich mich selbst überzeugte - ein sehr grazil gebauter, zarter Knabe, dessen Körperkräfte zu der geschilderten Wucht des Bürstenwurfes nicht recht passen wollen. Die Bürste selbst ist eine gewöhnliche, leichte, blob $200 \mathrm{gr}$. schwere, gestielte Teppichbürste ohne scharfe Kanten. - Auch die Pflegemutter der Pat. hat an der Verletzungsstelle ,nichts bemerkt".

Aus den Aussagen des zweiten Dienstmädchens und denen der Pflegemutter der Patientin geht mit Sicherheit hervor, daß sie sich 
schon lange vor dem Unfall nicht recht wohl fülte und damals schon schlecht ausgesehen habe. Sie habe schon Ende Juli über

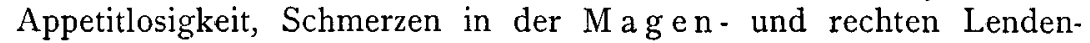
gegend, sowie über Schlaflosigkeit geklagt. Ende Juli habe sie -- während ihrer Ferienzeit - deswegen einen Arzt in B. konsultiert, der ihr gesagt habe, sie leide an "Wanderniere". -

Herr Priv.-Doz. Dr. K. in B. war so freundlich, mir darüber folgendes mitzuteilen: „Frl. B. H. stellte sich mir am 26. VII. I9I 5 zur Untersuchung. Sie klagte über Schmerzen in der Leber. ge gend vorn und hinten, die gegen Abend sich verstärkten. Ich fand normalen Genitalstatus; Leber scheinbar nicht vergrößert, darunter einen kleinen Tumor, der sich in senkrechter Richtung um ca. $4 \mathrm{~cm}$ verschieben ließ und der mir alle Zeichen einer Wanderniere darzubieten schien". - Dem betreffenden Arzt sei ebenfalls das schlechte Aussehen und ein damals schon mäßiger Ernährungszustand aufgefallen. -

Befund bei der Aufnahme am 7. X. 1915:

Mittelgroße, ziemlich grazil gebaute Patientin mit gut entwickelter, aber etwas schlaffer, welker Muskulatur und mäBigem Ernäh. rungszustand. -

Die Haut ist sehr blaß, zeigt keinerlei Exantheme, keine abnorme Pigmentierung, keine ikterische Verfärbung. Auch bei genauester Inspektion der ganzen Körperoberfläche finden sich keine Muttermäler oder Hautfibrome. Die Haut fühlt sich trocken an, ist nicht in Falten abhebbar. Es bestehen nirgends Hautödeme.

Temperatur in axilla a f e bril.

Pat. macht einen etwas müden Eindruck, antwortet aber lebhaft und zweckentsprechend auf alle Fragen. - Sie ist bei freiem Senso. rium, klagt über andauernde drückende oder dumpfbohrende Schmerzen in der Lebergegend, ohne daß sie dieselben genau lokalisierer. könne. Hie und da strahlen die Schmerzen ins Kreuz und nach den Schulterblättern zu aus, nie aber nach unten. - Gelb sei die Pat. bis jetzt nie gewesen. -

Kopf nach allen Richtungen hin frei beweglich, keine Nackenstarre. Haare schwarz, voll. Keine Narben, keine sonstigen Veränderungen in der Kopfschwarte. -

Gesichtsfarbe fahl, bleich, ungesund; das Gesicht als Ganzes ziemlich eingefallen. Keine Cyanose des Gesichtes. Augen blicken ziemlich matt, trübe. -

Die Skleren sind rein, weiß; die Conjunctiven beiderseits ziemlich blaß und anämisch.

Die rechte Pupille wird an ihrem Außenrand bedeckt von einer großen, weißen Macula, die sich noch über den Pupillarrand auf die Cornea fortsetzt und entsprechende Sehstörungen bedingt. Linkes Auge völlig normal; linke Pupille mittelweit, auf Lichteinfall gut 
reagierend. Der Augenspiegelbefund ergibt beiderseits heine Veränderungen des Augenhintergrundes. Die Regenbogenhaut beiderseits ohne makroskopisch sichtbare Veränderungen. Keine Doppelbilder. -

Nase und Ohren zeigen nichts Besonderes. Trommelfellbefund normal, Hammerreflex vorhanden. Pat. hört Flüstersprache auf $6 \mathrm{~m}$. Lippen nicht cyanotisch, etwas trocken und rissig. Kein Herpes labialis. Zähne zum größten Teil kariös. Zunge ziemlich stark belegt; starker Foetor ex ore. -

Im Rachen keine Rötung; Tonsillen von normaler Größe, ohne Beläge. Keinerlei Zeichen von Stomatitis. Rachenreflex vorhanden. Keine strahligen Narben im Rachen.

Der laryngoskopische Befund ist durchaus normal; Stimme klar.

Hals mittellang. Keinerlei Drüsenschwellungen zu konstatieren; Schilddrüse leicht vergrößert.

Thorax gut gebaut; beide Thoraxhälften von gleicher Größe und Weite. Druck auf den Thorax nicht empfindlich außer am rechten Rippenbogenrand. Über dem Manubrium sterni keine abnorme Dämpfung.

Atmung etwas beschleunigt und oberflächlich, vorwiegend kostal. Man hat den Eindruck, als ob die rechte Thoraxseite besonders in den vordern untern Partien etwas zurückbleibe. - Zeichen objektiver Dyspnoe sind nicht nachzuweisen. -

In den Supraclavicular- und Axillargruben keine Drüsenschwellungen.

Perkussion und Auskultation von Lungen und Herz ergeben durchaus normale Verhältnisse innerhalb normaler Grenzen. Nirgends Dämpfung. -- Die Lungen-Lebergrenze ist aber abnorm hoch (6. Rippe).

Wirbelsäule zeigt keine abnormen Krümmungen, keine Gibbus. bildung. Perkussion der einzelnen. Wirbel ist nicht empfindlich; Stauchungsschmerz fehlt vollständig. Brüste gut entwickelt, ohne. irgendwelche abnormen Verhärtungen oder Tumorbildungen.

A b domen: Die Unterbauchgegend ist überall weich, gut eindrückbar, auf Druck nicht empfindlich. Keine Muskelstarre. Nirgends eine Auftreibung; peristaltische Bewegungen nicht zu sehen. In der Unterbauchgegend ist eine abnorme Resistenz nicht zu fühlen. - Die Milz ist palpatorisch nicht zu fühlen; die Höhe der Milzdämpfung beträgt in der mittleren Axillarlinie $5-7 \mathrm{~cm}$ und geht vorn nicht über die Linea costoarticularis hinaus. -

In der rechten Oberba u ch ge gend fällt sofort eine ziemlich flache, aber deutliche Vorwölbung auf. - Sie erstreckt sich zum Teil über den Rippenbogen hinauf, geht nach innen bis fast zur Medianlinie, nach unten zwei bis drei Querfingerbreit vom Rippenkorbrande - Irgendwelche abnorme Verfärbung der rechten Ober- 
bauchgegend ist nicht zu konstatieren, dagegen eine deutliche Ektasie der Bauchhautvenen.

Bei der Palpation fühlt man deutlich einen ca. mannsfaustgroßen, unter dem rechten Rippenkorbrande hervorkommenden $\mathrm{T} u$ mo $r$ von prall-elastischer Konsistenz. Der untere Leberrand scheint in den Tumor überzugehen. Man hat auch entschieden den Eindruck, als ob der fragliche Tumor nach oben und seitlich, nicht aber nach unten verschiebbar sei. Die Palpation ist mäßig druckempfindlich. Leberdämpfung reicht von der 6. Rippe bis zwei Querfingerbreit unterhalb des Rippenkorbrandes. - .

Über den abhängigen Partien keine Dämpfung. Die Nieren, insbesondere die rechte, sind nicht palpabel, nicht druckempfindlich. Nieren- und Blasengegend o. B.

Dic Vaginal-Untersuchung ergibt normale Verhältnisse. -- Uterus und Adnexe o. B. Parametrien und Douglas frei. -

Weder Sensibilitäts- noch Motilitätsstörungen; Reflexe normal. - Die Extremitäten zeigen nichts Besonderes; insbesondere erweisen sich sämtliche Knochen auf Druck und Klopfen schmerzfrei. -

Urin klar, von strohgelber Farbe. Spez. Gewicht IOI 5. Er enthält weder Eiweiß noch Zucker; Hellersche Blutprobe negativ. Im Sediment keine Epithelien, keine Zylinder.

Io. X. Wassermann negativ. Die Blutuntersuchung (Privatdozent Dr. O. Rot h) ergibt:

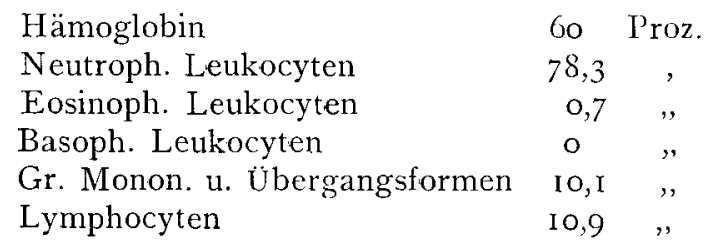

Starke Polychromatophilie. - Leukocytenzahl 8040.

Die Diagnose wird auf "Tumor hepatis", wahrscheinlich maligner Art gestellt und dabei ein Zusammenhang mit dem vorausgegangenen Trauma angenommen. - Der Patientin wird eine Probelaparotomie vorgeschlagen, in die sie einwilligt.

I3. X. Operation (Direktor Dr.Stierlin). Dauer 40 Min. Bromäthyl-Äther-Narkose, Ioo ccm. Jod-Alkohol-Desinfektion. Intrarektaler Schnitt rechts über die Kuppe des Tumors. Nachdem man den Rectus und die darunter liegende Fascie durchtrennt hat, kommt man auf die Tumoroberfläche, mit der das zarte Peritoneum parietale weithin fest verwachsen ist. Erst in Nabelhöhe kommt manı in die freie Bauchhöhle. Das Netz ist überall mit dem Tumor stark verwachsen. Nun wird das Colon transversum vorgezogen, ebenso der stark nach links verdrängte, mit dem 'Tumor durch feste Adhäsionen verbundene Magen. - Durch Palpation läßt sich nun 
feststellen, daß der Tumor dem sehr stark vergrößerten rechten L eberlappen angehört. - Er scheint über mannsfaustgroß zu sein, zeigt nirgends deutliche Fluktuation; man hat im Gegenteil überall das Gefühl von Spannung und Härte. In der U m g ebung des Tumors erscheint die Leber höckerig. Der Versuch, die Netzverwachsungen vom Tumor zu lösen, führt zu unangenehmer. schwer zu stillender Venenblutung. Der Tumor wird als inoperabel angesehen, die Operation abgebrochen und die Bauchhöhle wieder geschlossen.

I5. X. Pat. hat den Eingriff gut überstanden. Keine Temperaturerhöhungen.

20. X. Pat. bricht seit 3 Tagen anhaltend in kürzern und längern Abständen, trotz wiederholter Magenspülung, entsprechender Diät und Lagerung. - Das Erbrochene ist nicht blutig, riecht säuerlich. - Gesamtazidität 6o; freie $\mathrm{HCl} 0,05$.

25. X. Das Erbrechen hat aufgehört; Pat. fühlt sich nunmehr ganz ordentlich. - Nähte vor einigen Tagen entfernt. Wunde p. p. geheilt.

Es fällt auf, daß die Venektasien der Bauchhaut immer stärker werden Gesichtsfarbe womöglich noch blasser. Pat. klagt über dieselben Schmerzen in der Lebergegend wie vor der Operation.

30.X. Defäkation und Exurese immer geregelt. Bei wiederholter Urinuntersuchung kann nie EiweiB konstatiert werden; im Sediment vereinzelte Epithelien, aber nie Zylinder. Hellersche Blutprobe jmmer negativ. -

I 5. XI. Der Tumor wird immer größer; Umfang des Abdomens über dem Tumor gemessen $79 \mathrm{~cm}$. Abendtemperaturen i m m er lei cht erhöht.

Gewicht wie vor drei Wochen $53 \mathrm{~kg}$.

Röntgendurchleuchtung ergibt Hochstand des Zwerchfells rechts. Lungen zeigen keine Metastasenbildung.

23. X. Der Tumor scheint fast täglich größer $\mathrm{zu}$ werden, und zwar erfolgt die rapide VergröBerung deutlich in der Richtung von oben nach unten. - Die untere Tumorgrenze hat den Nabel bereits überschritten. - Umfang des Bauches $82 \mathrm{~cm}$. Gewichtsabnahme urm I $\mathrm{kg}$.

26. XI. Status idem. Bauchumfang $84 \mathrm{~cm}$.

I. XII. Zustand im ganzen derselbe. Es treten Atembeschwerden auf. Der Tumor wächst weiter; unterer Rand desselben handbreit unterhalb des Nabels. Bauchumfang $86 \mathrm{~cm}$.

5. XII. Man hat das Gefühl, als ob der Tumor nunmehr zum Teil fluktuiere. Deshalb wird unter aseptischen Kautelen eine Probepunktion vorgenommen; man erhält ca. $40 \mathrm{ccm}$ schokoladefarbene Flüssigkeit. 
Dic chemische Untersuchung der Punktionsflüssigkeit (Privatdozent Dr. E. Herzfeld) ergibt:

Reaktion: alkalisch.

Eiweiß: 5,86 Proz. (Mucoide, Albumin, Globulin).

Harnst off : schwach positiv.

Harnsäure: schwach positiv.

Traubenzucker: Spuren.

Bilirubin: positiv.

Urobilin : negativ.

Blut: stark positiv.

Cholesterin: positiv.

Fett: positiv.

Bernsteinsäure: negativ.

Die bakteriologische Untersuchung der Punktionsflüssigkeit ( $\mathrm{Hy}$ giene-Institut der Universität Zürich, Direktor: Prof. Dr. Silber$\mathrm{schmidt}$ ) ergibt in Agar und Bouillon kein Wachstum; Mikroorganismen nicht nachweisbar. -

I3. XII. Haut und sichtbare Schleimhäute werden blasser. Hämoglobingehalt 55 Proz. (S a h li). Die Punktion wird noch einmal genau an derselben Stelle wie früher wiederholt; es gelingt diesmal nicht, Flüssigkeit aufzusaugen. - Man hat das Gefühl, die Nadel stecke in festem (Leber?-) Gewebe und nicht in einem Hohlraum. -

Wiederholt therapeutische Röntgenbestrahlung (Je 70 Kienböck-E.)

28. XII. Pat. hat wenig Beschwerden und ist in einer auffallend euphorischen Stimmung. Leibesumfang $86 \mathrm{~cm}$. Die Bauchhaut-Venenerweiterung wird immer stärker ausgeprägt. Herr Prof. $\mathrm{S}$ a u e r bruch, der die Patientin sah, hält die Diagnose eines Leber$\mathrm{S}$ a rkoms für wahrscheinlich.

4. I. I6. In den letzten I 4 Tagen ist der Tumor ganz beträchtlich gewachsen; der untere Leberrand ist nunmehr nicht ganz Handbreit über der Symphyse. Puls stark beschleunigt, hie und da unregelmäBig. Wohl als Kompressionserscheinung des Magens erklären sich häufige Übelkeit und häufiges Erbrechen.

I5. I. Leibesumfang nunmehr I Io $\mathrm{cm}$.

Es beginnen sich immer deutlichere Ascites-Symptome, auch zunehmende Ödeme an den Oberschenkeln einzustellen. Die Vorwölbung der Oberbauchgegend hat monströse Formen angenommen (Fig. I).

Fortwährende Abmagerung; starkes Druckgefühl und Schmerzen in der Lebergegend. Atmung immer oberflächlicher und mühsamer. Das Zwerchfell steigt - perkutorisch deutlich nachweisbar .-- auf der rechten Seite immer mehr in die Höhe.

Herz nach links verlagert. Spitzenstoß zweiquerfingerbreit außerhalb der Mamillarlinie. - Auskultation und Perkussion der Lungen 
ergeben außer vesico-bronchialem Atemgeräusch über dem rechten Unterlappen nichts Besonderes. -

Abendtemperaturen schwanken zwischen $37, \mathrm{I}-38,2$ Grad.

I. II. 16. Allgemeinbefinden immer ganz ordentlich. Die stark euphorische Stimmung der Patientin hält an. In frappantem Gegensatz dazu steht die deutlich fortschreitende Abmagerung bei immer größer werdendem Abdomen. Lippen cyanotisch. Puls zwischen I 40 bis I 50, sehr weich, inäqual und unregelmäßig trotz fortwährender Digalen-Medikation.

Defäkation und Exurese geregelt. Im Urin sind bei wiederholten Untersuchungen nie Eiweib oder Zucker gefunden worden. Keine Lävulosurie. Kein Urobilin, kein Blut im Urin. Diazoreaktion stets negativ. Im Sediment können Zylinder nie gefuuden werden.

I. III. Fortschreitender Zerfall. Palpatorisch läßt sich feststellen, daß der Tumor seitlich und nach unten nunmehr das ganze Abdomen ausfüllt. Daneben starker Ascites, so daß der Bauch enorm gespannt crscheint. Starke Ödeme an den Beinen. Leibesumfang $120 \mathrm{~cm}$.

Pat. nimmet in den folgenden 3 Wochen fast nichts mehr zu sich, schläft viel. Unter den Erscheinun. gen zunchmender Herzschwäche Exitus letalis am I5. III. I9I6.

Autopsic I6. III. I9I6. Obduzent: Prof. Dr. O. Busse, Direktor des patholog. Instituts der Universität Zürich.

Kleine untersetzte weibl. Leiche, bei der eine geradezu unge-

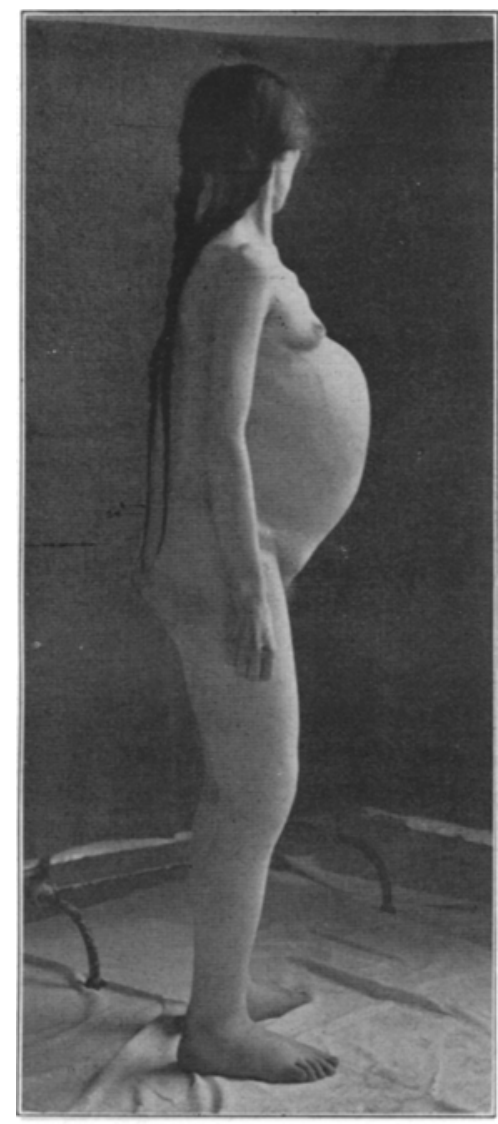

Fig. I. heuerliche Auftreibung des Leibes vorliegt, und zwar in der oberen Hälfte. Der Leibesumfang beträgt hier $126 \mathrm{~cm}$. Auf der Mittellinie des Bauches verläuft eine Narbe von $22 \mathrm{~cm}$ Länge. Sehr starke Entwicklung der Venen. Im untern Teil des Bauches sehr starkes Ödem, das auch die untern Extremitäten unförmig gestaltet hat. 
Mit großer Vorsicht wird der Bauch eröffnet. Dabei läßt sich eine Trennung zwischen Tumor und Bauchdecken nicht herbeiführen. Der Schnitt geht durch eine dünne Stelle des Tumors hindurch und unter hohem Strahl und starkem Druck entleert sich rötlichgelbe Flüssigkeit. Es lassen sich allmählich von dieser Flüssigkeit gröbere Mengen auffangen, die schließlich $2400 \mathrm{ccm}$ betragen. Dann kommt man tief unterhalb des Nabels in die freie Bauchhöhle und hier entleert sich reichlich milchige Flüssigkeit aus dem Bauche, von der $2200 \mathrm{ccm}$ aufgefangen werden. Ein Teil dieser Flüssigkeit liegt auch, wie sich nachher zeigt, zwischen Leber und $r$. Zwerchfell. Die Geschwulst wächst in die Bauchdecken hinein, und diese sind an tinzelnen Stellen so dünn, daß tatsächlich nur eine ganz minimale Lage von kaum mehr als I $2 \mathrm{~mm}$ Dicke über die Geschwulst hinwegzieht. Nachdem mit großer Mühe ein Teil der Geschwulst freigelegt ist, läßt sich die Haut, die diffus mit der Oberfläche des Tumors verwachsen ist, doch teils stumpf, teils scharf davon trennen. Die Geschwulst überragt das Sternum um ca. $32 \mathrm{~cm}$. Das $Z_{\text {werchfell ist }}$ rechts bis in den 2. Interkostalraum nach oben gedrängt, während es links am untern Rand der 5. Rippe steht. Die Geschwulst wächst breit aus der Leber, und zwar aus dem rechten Lappen beraus. Am Lig. suspensorium hört der Tumor auf und der linke Leberlappen sitzt wie ein kleines Anhängsel an der Geschwulst. Die obere Kuppe des r. Leberlappens ist vollkommen frei und verhältnismäßig weich, und erst nach unten geht dann die Leber mehr und mehr in clie Geschwulst über, so daß das Lebergewebe immer mehr verdünnt wird.

Es wird nun der Tumor isoliert. (Fig. 2.) Das Netz ist überall mit dem Tumor verwachsen, so daß die Geschwulst hier scharf davon befreit werden muß. Dabei sind nach und nach die Schichten des Colons in die Geschwulst derart aufgegangen, daß schließlich nur dic Schleimhaut als eine dünne, durchsichtige Membran abgelöst wird, die aber auch noch einreißt. Die linke Nicre steht in keinem Zusammenhang mit der Geschwulst, ebenso läßt sich die Milz leicht ablösen. Der Magen ist ganz nach links hinübcrgedrängt und steil gestellt, so daß die große Kurvatur an der linken Bauchseite ron oben nach unten zieht. Die kleine Kurvatur ist durch das Lig. hicpatogastricum mit der Leber und dem Tumor verbunden, läßt sich aber leicht abtrennen, und nun sieht man in der Tiefe das breite, lappige Pankreas, das sich hart anfühlt. An der Pars pylorica ist die klcine Kurvatur des Magens dem Tumor adhärent. Ebenso ist das Duodenum damit verwachsen derart, daß eine Trennung nicht möglich ist. Dic rechte Niere ist zunächst nicht klar herauszufinden, weil an der Geschwulst eine Anzahl von Knoten und Protuberanzen hervortreten; diese Vorsprünge sind zum Teil crweicht und mit Flüssigkeit gefüllt. - Es wird deshalb beschlossen, die Geschwulst mit den Nieren, der Aorta, dem Magen und Duodenum im Zusammenhang 
zu belassen. Dazu wird schnell die Brusthöhle ausgeräumt. Die linke Brusthöhle enthält etwa $600 \mathrm{ccm}$ blutiger Flüssigkeit; die rechte ist verödet.

Im Herzbeutel $30 \mathrm{ccm}$ Flüssigkeit. Das Herz ist vergrößert, der rechte Ventrikel dilatiert, auch etwas hypertrophiert. Das linke Herz ist kräftig, der Ventrikel $9 \mathrm{~cm}$ lang, die Aorta $7,8 \mathrm{~cm}$ breit. Die Muskulatur des Herzens ist transparent mit leichten Flecken.

Die linke Lunge läßt sich leicht herausnehmen, sie ist pigmentfrei, der Unterlappen stark hypostatisch und atelektatisch. Der Oberlappen ist lufthaltig.

Dic rechte Lunge muß mit der Pleura cost. herausgenommen

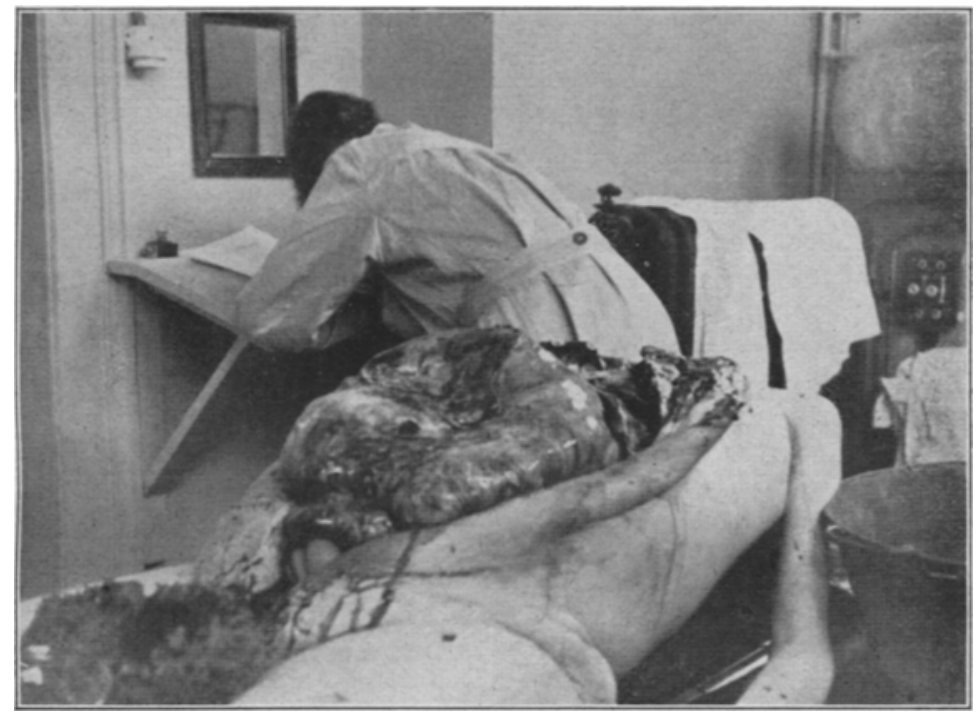

Fig. 2.

werden. Der Unter- und Mittellappen sind hypostatisch und fester. Der Oberlappen ist lufthaltig.

Nun wird mit einigen Schwierigkeiten der Tumor mit den umliegenden Teilen aus der Bauchhöhle entfernt. Der Magen wird aufgeschnitten. Er enthält sehr viel Speisebrei, Gemüsereste und dergl. Im Duodenum etwas galliger Inhalt. Jetzt wird eine Sondierung der Papille vorgenommen, die in den Ductus pancreaticus führt. Auch der Duct. choledochus wird sondiert. Beim Sondieren und Aufschneiden kommt man schließlich in einen Gang, der stark komprimiert mit klappenartigen Leisten versehen ist und sich schließlich nicht weiter verfolgen läßt. Offenbar ist dies der Duct. cysticus. 
Der Duct. hepaticus läßt sich mit seinen Ästen bis in die Leber hinein verfolgen, er ist mit Galle angefüllt und stark verlängert, so da $B$ die Entfernung vom Duodenum bis zur Leber hin etwa $17 \mathrm{~cm}$ beträgt. Die Gallenblase ist nicht aufzufinden.

Die Geschwulst mißt von der Leber bis zum untern Rande $30 \mathrm{~cm}$, sie ist auch ca. $30 \mathrm{~cm}$ breit und hat eine Dicke von $25 \mathrm{~cm}$.

Die linke Niere ist vollkommen frei, sie mißt Io:8:3 cm. Ihre Oberfläche ist glatt, die Rinde transparent.

Die rechte Niere ist zu beiden Seiten des oberen Pols von einer polsterartigen Geschwulstmasse umgeben, der untere Pol der Niere ragt daraus hervor. Beim Aufschneiden sieht man nun, daß der Hilus der Niere durch eine unter gröBerer Spannung stehende Tumormasse von mehr als Faustgröße eingenommen wird, welche die Niere über sich gezogen und gedehnt hat, so da $\beta$ der obere Pol bis auf $3 \mathrm{~mm}$ verdünnt ist, während der untere Pol deutlich Rinde und Mark erkennen läßt und auch Nierenbecken und Kelche deutlich hervortreten. Der Durchschnitt mißt hier bis zu $4 \mathrm{~cm}$.

Es wird nun durch den Tumor ein Schnitt derart gelegt, daß der rechte Leberlappen von oben nach unten her halbiert ist; der Schnitt läuft also parallel zur Längslinie des Körpers. Auf diesem Durchschnitt stellt sich die Geschwulst aus zwei Hauptteilen bestehend dar, einer größern, soliden Hälfte, die der Leber angrenzt und einer zweiten Hälfte, die zum Teil cystisch degeneriert gegen das Becken hingeht. (Fig. 3.)

Die Geschwulst im ganzen mißt jetzt auf dem Durchschnitt $35 \mathrm{~cm}$ in der Länge und etwa $25 \mathrm{~cm}$ in der Breite. Die obere, solide Geschwulst hat eine Länge von etwa $25 \mathrm{~cm}$; sie grenzt in bogenförmiger Linie gegen die Leber derart, daß ein $10 \mathrm{~cm}$ breites Stück der Leber d $\epsilon r$ Geschwulst aufsitzt, und in einem zungenförmigen Streifen an der Vorderfläche der Geschwulst herunterzieht, bis er - immer dünner werdend - schließlich ganz verschwindet. Die Schnittfläche der Geschwulst ist im ganzen glasig, graurot, auch gelblich gefärbt, doch so, daß sich einzelne Knollen und Lappen noch deutlich und besonders vorwölben. Die Konsistenz ist prall elastisch; einzelne Partien sind sehr viel weicher als einzelne derbere Abschnitte. Die untere Hälfte der Geschwulst wird von der großen Erweichungscyste eingenommen, aus der die anfangs erwähnten $2400 \mathrm{ccm}$ Flüssigkeit stammen. Die Wandungen der Cyste sind zerfetzt, zum Teil von Fibringerinnseln gebildet und sehr verschieden dick; an einzelnen Stellen bis $8 \mathrm{~cm}$, an andern wieder nur $2-3 \mathrm{~mm}$. Weiche, nekrotische Fetzen ziehen durch die Cyste hindurch. Die Cyste weist demnach Ausbuchtungen und Nischen der verschiedensten Art auf. Bei allem Suchen ist aber auch jetzt eine Gallenblase nicht zu finden.

Metastasen können in keinen Organen der Brust- oder Bauchhöhle gefunden werden, ebensowenig im Knochensystem. 
Die Beckenorgane bieten keine Besonderheiten dar. Der Uterus ist verhältnismäBig klein; die Ovarien sind narbig und schon stark geschrumpft. Die Harnblase ist leer. Im ganzen Darm ist gallig gefärbter Inhalt in mäßiger Menge.

Für die mikroskopische Untersuchung werden Stücke aus den verschiedenen Stellen der Geschwulst eingelegt. Es zeigt sich in mikroskopischen Präparaten, daß die Geschwulst übereinstimmend in allen Teilen aus einem zellenreichen sarkomatösen Gewebe besteht. Die Zellen wechseln in ihren Formen. In großen Ab-

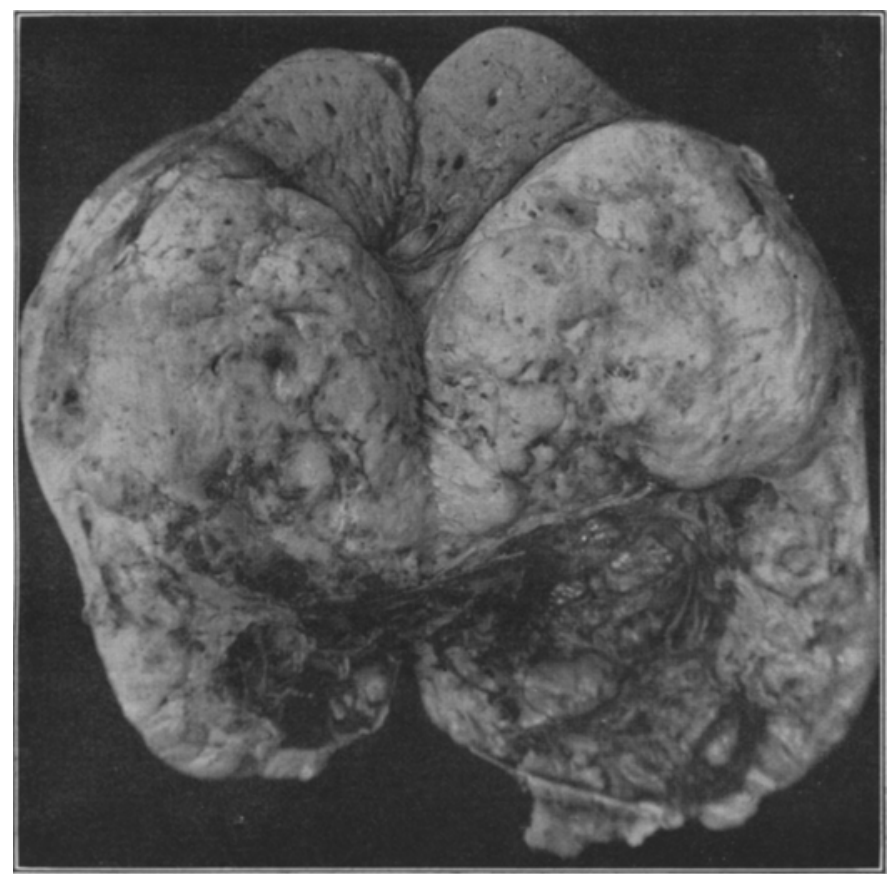

Fig. 3.

schnitten der Geschwulst überwiegen die Spindelzellen, die vielfach in Zügen und Reihen angeordnet sind und zwischen sich feinste Fibrillen von der Interzellularsubstanz fassen. An andern Stellen findet sich ein weiches, körniges Material zwischen den Zellen, und hicr sieht man auch, daß die Zellen vielfach ihre Form verändern, und vor allen Dingen an Größe zunehmen, so daß dann saftreiche, mit Ausläufern versehene Spindel- und Sternzellen zu finden sind. Unter dicsen zeiger: sich dann immer vereinzelte Zellen, dic durch ihren großen Kern auffallen. Diese Kerne haben gewöhnlich auch stark den Farb- 
stoff zurïckbehalten und treten schon so bei schwacher Vergrößerung als dunkle klecksige Gebilde hervor, deren Größe die der gewöhnlichen Kerne um mehrfaches übertrifft. An andern Stellen sind diese größeren protoplasmatischen Zellen mit mehreren Kernen ausgestatter, so dal. sic eine gewisse $\ddot{A}$ hnlichkeit mit Riesenzellen bckommen. Noch andere Abschnitte enthalten zahlreiche kleine rundliche Formen, ohne eine bestimmte $\Lambda$ nordnung. Somit zeigt sich also, da $\beta$ hier tine ex. quisit sarkomatöse Geschwulst vorliegt, deren Zellen im wesentlichen Spindelformen tragen, zum Teil auch allerdings andere Formen erkennen lassen.

Nachträglich werden vom gehärteten Präparat noch einige Stückchen abgeschnitten, die von der Grenze des Tumors zur Leber stammen. Bei der mikroskopischen Untersuchung dieser Stücke sieht man, dafü zwischen der durch den Tumor komprimierten Leber und der Geschwulst eine breite, kernarme Bindegewebskapsel zieht, durch die Geschwulst und Leber getrennt sind. Unmittelbar an dieser Kapsel liegen die großen saftreichen Geschwulstzellen mit einem sehr weichen Zwischengewebe. Andere Schnitte zeigen an der Grenze von Leber und Geschwulst einen höckrigen lappigen Bau des Tumors und man sieht nun wie die Kapsel auch als bindegewebiges Septum zwischen die verschiedenen Lappen der Geschwulst zieht. Hier allerdings zeigt sich dann, da $B$ dieses zwischen den Lappen gelegene Septum vielfach sehr zellenreich ist und auf einer Seite wenigstens ganz allmählich bei Zunahme des Zellenreichtums in die Sarkomgeschwulst übergeht.

Dic Leber selbst ist im großen und ganzen unverändert, insonderheit frei von interstitiellen Bindegewebswucherungen. Nur die an die Geschwulst grenzenden Bezirke zeigen Kompression der Zellen und Lobuli infolge des Druckes, den die Geschwulst ausgeübt hat. Pathologisch-anatomische Diagnose:

Sarcoma permagnum fusi-cellularelobidext. hepat is prim., hilum renis dext. infiltrans. Hydrops-Ascites chylosus.

Von Cornil und Ranvier, die das Vorkommen primärer Lebersarkome überhaupt bestritten, bis zu dem Erscheinen der zusammenfassenden Monographie von Thöle, liegt cin Zeitraum von fast 30 Jahren. - Aber alle Statistiken und kasuistischen Beiträge haben nicht vermocht, unsere Kenntnisse über die klinischen Erscheinungen so zu bereichern, daß Diagnose und Therapie früher möglich, die Prognose des primären Lebersarkoms dementsprechend besser geworden wäre. - Auch heute noch gilt für die Klinik des primären malignen Lebertumors der 
resignierte Satz Bertellis, daß das klinische Bild keine für die Erkrankung charakteristischen Symptome e rkennen lä $\beta$ t. Nicht tröstlicher ist der Ausspruch. Th öles, daßs die radikale Entfernung primärer, maligner Lebertumoren mittels ausgedehnter Leberresektion nur im Begin $n$ der Erkrankung möglich sei, wenn er selber hinzufügt, daß sie dann bis jetzt eben nicht diagnostizierbar seien. Die Therapie hat sich demgemäß bisher in allen Fällen als machtlos erwiesen - eine scheinbare Ausnahme, die $\mathrm{Kn}$ ot t publiziert, ist zu unvollständig, um beweisend zu sein. - ,Die erfolgreiche Exstirpation cines primären Lebersarkoms wird vorläufig ein glücklicher Zufall bleiben, herbeigeführt durch eine frühzeitig auf Grund von Verdachtsmomenten vorgenommene Probelaparotomie“. (Thöle.)

Die Anfangssymptome waren auch in unserem Falle ganz unbestimmte und versteckten sich unter den Erscheinungen von Magen-Darmstörungen, wie dies auch $\mathrm{Sch}$ eidemandel, Thöle, Orth u. a. in ihren Fällen beobachtet haben.

Der für Leberaffektionen einigermaßen charakteristische $\mathrm{Schulterschmerz}$ war angedeutet vorhanden; dagegen fehlten Ikterus und - wenigstens im Anfang - Ascites. Das F e hl en von Ikterus und Ascites ist in den bis jetzt in der Literatur bekannten Fällen so allgemein, daß Thöle darauf in der Diagnose der malignen Lebertumoren ein großes Gewicht legt. Der enormen Bauchhautvenenerweiterung, wie sie auch in unserem Falle bestand, wird von den meisten Autoren kein besonderer diagnostischer Wert zugemessen. Erweiterung der Bauchvenen ist bei großen Lebertumoren leicht verständlich; ihr aber eine differentialdiagnostische Bedeutung zuerkennen zu wollen, geht nicht an. -

Die von verschiedenen Autoren beobachtete Temperatursteigerung bei Sarkomen war auch hier vorhanden; dagegen f e hlte Leukocytose und Eosinophilie. Kast (zit. nach Thöle) wollte das Sarkomfieber mit Metastasenbildung in hämatopoetischen Organen in Zusammenhang bringen, eine Annahme, deren Unrichtigkeit verschiedentlich betont und auch durch unsere Beobachtung widerlegt wird. -

Gestützt auf die Anamnese, von der weiter unten noch zu sprechen sein wird, und den bei der Aufnahme erhobenen klinischen 
Befund wurde die Diagnose auf "maligner Lebertumor" gestellt, die Entscheidung zwischen Sarkom und Carcinom offen gelassen. Das Einzige, was eventuell eher für Sarkom gesprochen hätte, wäre das jugendliche Alter und das nach der Darstellung der Patientin sehr rasche Wachstum des Tumors gewesen. -

Der 'Tumor selbst wurde nach dem erhobenen klinischen Befund von Anfang an als der Leber angehörend betrachtet. Konnte man erst über die Natur des festgestellten Lebertumors im Zweifel sein - wir dachten erst an einen abgekapselten intrahepatischen, traumatischen BluterguB, auch der Gedanke an ein Zystadenom der Leber wurde erwogen - so mußten die deutlich in Erscheinung tretende Abmagerung, das rasche Größerwerden des Tumors bald dic Malignität desselben erkennen lassen. Für Leberechinococcus lagen in Anbetracht der Anamnese und des hierzulande äußerst seltenen Vorkommens keinerlei Anhaltspunkte vor. -

Der Tumor wurde klinisch als primär angesehen, da sich keinerlei andere Tumor- oder Metastasenbildungen nachweisen ließen.

Garbarini (zit. nach 'Thöle) hält eine Differentialdiagnose zwischen Sarkom und Carcinom nach folgendem Schema für möglich:

Sarkom

I. I.eber sehr vergrößert, Oberfläche eben oder bucklig, aber glatt.

2. Ikterus und Ascites selten, nicht hochgradig.

3. Fieber oft, hoch, oft kontinuierlich.

4. Milz fast immer normal.

5. Urin vermindert, selten Gallenfarbstoff.

6. Symptome von Acholie.

7. Rascher akuter Verlauf.

8. Meist bei jüngern Individuen und Kindern.
Karzinom

I. Leber normal groß oder etwas vergrößert, hart, bucklig oder granuliert.

2. Ikterus und Ascites oft und ausgesprochen.

3. Fieber selten und nicht hoch.

4. Milz oft hyperplastisch.

5. Urin reichlich, oft Gallenfarbstuff.

6. Symptome von Gallenretention.

7. Verlauf langsam, chronisch.

8. Meist bei Erwachsenen und ältern, ausnahmsweise bei jüngern Individuen.

Inwieweit dieses Schema für den e in zelne n F a 11 anwendbar ist, möge dahingestellt bleiben; Thöle spricht ihm jeg. liche Bedeutung ab.

Auch nach der Probelaparotomie waren wir nicht in der Lage, 
mit Sicherheit Sarkom festzustellen, analog den Fällen von $M$ ü ller, Calvini, Kümmell und Garrè (zit. nach Thöle), und erst die Autopsie und die mikroskopische Untersuchung haben die klinische Diagnose genauer spezifiziert.

Bei der Probelaparotomie erwies sich die Geschwulst kereits als zu ausgedehnt, die höckrige Beschaffenheit des angrenzenden Lebergewebes lie $\beta$ bereits zu sehr Metastasierung und Rezidivgefahr fürchten, als daß eine operative Entfernung noch in Frage gekommen wäre. Sie hätte nur in totaler Resektion des rechten Leberlappens bestehen können.

Daß selbst Resektionen ganzer Leberlappen technisch und physiologisch möglich sind, ist seit der Veröffentlichung Li u s-E s chers im Jahre $\mathbf{1} 886$ bekannt. Technisch ist ja die Möglichkeit der Resektion nicht anderes als die Möglichkeit der Blutstillung. Es ist hier nicht der Ort, über alle die verschiedenen im Laufe der Zeit zur Anwendung gelangten Methoden der.Blutstillung sich auszulassen; hingewiesen sei immerhin auf neuere und von $\mathrm{T} h \mathrm{~h}$ ol e noch nicht berücksich. tigte Versuche von $\mathrm{Negri}$.Kornew und $\mathrm{Schaack}$, Chessin, Opokin und $\mathrm{Sch}$ a mof $\mathrm{f}, \mathrm{H}$ ilse.

N e gri resezierte nach dem Bauchdeckenschnitt ein Stück Peritoneum mit einer Schicht Muskulatur und bewahrte es in physiologischer NaCl-Lösung auf. Nach der Leberresektion - während provisor. Blutstillung durch manuelle Kompression - wird der Lappen in den Leberdefekt eingenäht. In to Tierversuchen beobachtete Negri, daß dieser muskulo-seröse Lappen ein sehr gutes Mittel zur Blutstillung darstellt.

In Nachahmung von Kirschner und Henschen haben Chessin und Kornew und Schaack das Prinzip der freien Fascientransplantation, das schon von Kirschner selbst auf die Leber angewendet wurde, in Einzelheiten modifiziert.

Opokin und Schamoff haben durch aufgenähte Muskelstücke Blutstillung erzielt. Das Muskelgewebe soll dabei nicht mechanisch, sondern in erster Linie durch seinen Gehalt an Thromb o k in a s e biologisch wirken. -

Hilse will durch frei transplantierte Fettlappen, die er auf der Resektionsfläche ausbreitete, in zahlreichen Tierversuchen und in zwei Fällen von Leberbettblutung nach Gallenblasenexstirpation eine vollständige Blutstillung erzielt haben.

So schön und interessant sich alle diese Versuche im Tierexperiment und der Beschreibung ihrer Autoren ausnehmen -- die systematische intrahepatische Massenligatur nach K ous net $\mathrm{z}$ of $\mathrm{f}$ und Pensky hat durch sie noch nichts von ihrer besonders von Thöle hervorgehobenen Bedeutung verloren. 
$\mathrm{DaB}$ auch operativ gelungene Resektionen bei malignen Lebertumoren bis jetzt keinen Da u ererfolg erzielt haben, ist leider nicht zu bestreiten. Vereinzelte scheinbare Ausnahmen, so die von Knott, Calvini, Mazzoni (l. c.) publizierten, sind in ihren Angaben zu ungenau oder unvollständig, um beweisend zu sein. -

II a sler z. B. glaubt nicht an irgendwelchen Dauererfolg bei Resektion einer Lebergeschwulst.

Was die operative Prognose der partiellen Leberresektion überhaupt anbetrifft, so gibt Thöle eine absolute Mortalität von 16,8 Proz. und eine relative - nur der Operation an der Leber als solcher zur Last zu legende - von 6,5 Proz. an, mißt aber selber dieser Berechnung nicht allzu großen Wert bei.

Dic operative Prognose der malignen Lebertumoren im besonderen ist, wie bereits erwähnt, eine ganz traurige. Das beweist auch eine Statistik Thöles von I4 Sarkomfällen:

Fall d'Antona: Tod am 2. Tag im Operationsshock.

Fall $\mathrm{B}$ arde $\mathrm{leben}$ (in die Leber eingewachsenes Bauchdeckensarkom): Nach 2 Jahren noch gesund. Endresultat nicht angegeben. Jahren.

Fall v. Beck: Tod an Leber- und Milzmetastasen nach $1 \frac{1}{2}$

Fall $\mathrm{C}$ alvini (gestieltes Sarkom): Operative Heilung in 4 Monaten. Endresultat nicht angegeben.

Fall Cle menti (eingewachsenes Mesokolonsarkom): Operative Ileilung. Tod nach 6 Monaten an Rezidiv.

Fall Czerny (Lebermetastasen bei Myosarcoma uteri): Tod am folgenden Tag an Peritonitis.

Fall Elliot (gestieltcs Sarkom): Operative IIeilung. Tod nach 3 Monaten an Rezidiv.

Fall Is rae1: Operative Heilung. Nach $3^{1 / 2}$ Monaten Tod an allgemeiner Sarkomatose.

Fall Knott: Operative Heilung. Endresultat nicht angegeben.

Fall $\mathrm{K}$ ü m mell (hasehußgroßes Sarkom am Leberrand): Operative Heilung. Endresultat nicht angegeben.

Fall Mazzoni: Durch Pneumonie gestörte operative Heilung. Endresultat fehlt.

Fall M üller: Operative Heilung. Nach 9 Monaten Tod an Re zidiv und Metastasen.

Fall Sklifasowsky: Operative Heilung. Endresultat fehit.

Fall Torrance: Resektion des ganzen linken Lappens. Tod nach 24 Stunden. - 
In Anbetracht des bei der Probelaparotomie erhobenen Befundes, der eine radikale Entfernung von vornherein als aussichtslos erscheinen ließ, hat Herr Direktor Dr. Stierlin auf den Versuch der operativen Entfernung von Anfang an verzichtet. - Auch Thöle betont, daß - wenn man bereits auch nur eine kleine Metastase befürchten müsse - die Resektion kcinen Sinn mehr habe. -

Grobanatomisch handelt es sich in unserem Falle um ein Sarcoma tuberosum im Sinne Thöles, das eine ganz gewaltige Größe erreicht hatte. - Farbe auf dem Durchschnitt graurötlich bis weißgelblich. In Anbetracht des geringen Gefäßreichtumes ist anzunehmen, daß der Ausgangspunkt wahrscheinlich das periphere Bindegewebe gewesen ist. Die Geschwulst wurde auch in unserem Falle durch Reste hyperplastischen Bindegewcbes in Felder geteilt, wie bei den Beobachtungen von $\mathrm{Ka} \mathrm{hl}$. den, de Vecchi, Carmichael and Wade (zit. nach I'höle).

Histologisch geht aus dem mikroskopischen Befund die Natur des Tumors als Spindelzellensarkom mit sehr spärlichem Stroma ohne weiteres hervor. Erwähnenswert ist der Umstand, daß sich eine große $\mathrm{C} y \mathrm{ste}$ im unteren Teil des Tumors fand. Es zeigt sich hier eine große Ähnlichkeit mit dem Befunde, den B ramwell erhob. Durch anämische oder hämorrhagische Nekrose können Sarkome im Zentrum zerfallen; es. entstehen eine oder mehrere Erweichungscysten. Die schokoladefarbige Flüssigkeit, die bei wiederholten Punktionen entleert werden konnte, stammt offenbar von einer Blutung in dieser Cyste her, iber deren Zusammenhang mit dem anamnestisch angegebenen Trauma noch zu sprechen sein wird. -

Einc Zunahme des elastischen Fasergewebes war nicht vorhanden. Cignozzi hält diese Tatsache für differentialdiagnostisch wichtig gegenüber den Lebercarcinomen, die eine starke Vermehrung der elastischen Fasern aufweisen.

Metastasen haben sich bei der Autopsie nach genauester Untersuchung keine gefunden. - Ebensowenig irgendwelche andern Tumorbildungen. Gehirn und Skelettsystem wurden allerdings nicht seziert; der Einwand, daß vielleicht ein primäres, aber latentes myelogenes Sarkom der Röhrenknochen oder Wirbel, 
oder ein Gliosarkom vorhanden gewesen sei, kann aber doch wohl nicht erhoben werden, da derartige Tumoren bekanntlich sehr frühzeitig Symptome machen, die klinisch eben nie vorhanden waren. - Auch ist wohl nicht anzunehmen, daß sie während ihrer Latenzzeit schon eine so große metastatische Erkrankung der Leber herbeigeführt hätten. - Es dürfte sich so ohne $Z_{\text {weifel um }}$ ein primäres Spindelzellensarkom der Leber han. deln.

Zu erörtern wäre noch der Umstand, daß bei der Autopsie trotz genauestem Suchen die Gallenblase nicht gefunden werden konnte, sondern im Tumor aufgegangen war. - Die Annahme, daß es sich demgemäß event. um ein primäres $G$ allenblasensarkom handeln könnte, ist nicht von vornherein von der Hand zu weisen, erscheint aber in Anbetracht der gewaltigen Größe und der histologischen Struktur des Lebertumors doch als unwahrscheinlich.

Thöle hat I9r 3 aus der gesamten Literatur Io Fälle von primärem Sarkom der Gallenblase zusammengestellt, $\mathrm{S} \mathrm{chön} \mathrm{lank}$ berichtet I9I4 über eine weitere Beobachtung aus dem pathologischen Institut der Universität Zürich, so daß bis jetzt bloß I I sicher primäre Sarkome der Gallenblase beschrieben sind. - Die Literatur findet sich zusammengestellt bei $\mathrm{H}$ otes, $\mathrm{L}$ and ste in er, $\mathrm{B}$ a y e r und $\mathrm{T} h \ddot{\mathrm{l} l \mathrm{e}}$.

In keinem dieser Fälle war ein derart riesiger Lebertumor neben dem Gallenblasensarkom vorhanden, wie er - unter dieser Annahme - bei unserer Patientin vorhanden war. Deshalb darf wohl angenommen werden, da $\beta$ Gallenblasensarkome nicht über einen gewissen, mittleren Umfang hinaus gehen und nur kleinere Lebermetastasen machen, ein Grund mehr für unsere Annahme eines primären L e b e r und nicht eines primären Gallenblasensarkoms.

In unserem Fall fehlten ferner Gallensteine vollständig, während für alle Fälle von primärem Gallenblasensarkom das Vorhandensein von Gallensteinen ausdrücklich hervorgehoben wird. Die meisten der Fali Schönlank ausgenommen - zeigten außerdem Metastasen in den verschiedenen Organen der Körperhöblen. - Die Größenverhältnisse in den beiden Beobachtungen Bayers (zit. nach Schönlank) waren $12: 9: 8$ und 19:14:6.

Operativ angegangen - unter der Diagnose Cholelithiasis wurden nur die Fälle v. Beck (zit. nach Thöle) und BrunnerSchönlank. Becks Pat. war nach Io Jahren noch gesund; dic Pat. Brunners starb ca. $22^{1 / 2}$ Wochen p. op. an multiplen Lungen embolien. 
Die richtige Diagnose ist ante operat. nie gestellt worden (Thöle).

Es sei mir nun noch gestattet, etwas näher auf die $\ddot{A}$ t iolo gie des Lebersarkoms in unserem Falle einzugehen. Die in der Krankengeschichte ausführlich angegebene Anamnese scheint mir deswegen nicht ohne einiges Interesse $z$ u sein, weil sie einen eigenartigen Beitrag zur Frage der traumatischen Entstehung der Sarkome darstellt. - Es kann selbstverständlich nicht meine Absicht sein, zu der vielumstrittenen Frage, die eine fast unabsehbare Literaturfülle gezeitigt hat, auf Grund dieses einen Falles Stellung nehmen zu wollen.

Lehrreich ist unsere Beobachtung aber doch, weil sie zeigt, wie leicht man sich in der Wertung eines Sarkomfalles ,als durch Trauma entstanden" täuschen kann.

Stellt man - wie wir es anfangs zu tun geneigt waren - nur auf die Angaben der Patientin ab, die an sich durchaus vertrauenswürdig und glaubhaft erschienen, so mußte man unbedingt zu der Auffassung kommen, daB - wenn überhaupt - in diesem Falle das stattgehabte Trauma die Entstehung des (Leber-)Sarkoms ursächlich bedingt habe. Ein nach seiner wiederholton Versicherung bis zum Zeitpunkt des Unfalls durchaus gesundes und voll arbeitsfähiges Dienstmädchen erhält einen heftigen Schlag in die Lebergegend, wird ohnmächtig und verspürt heftige Schmerzen, die seither beständig anhalten. Der I 4 Tage nach dem Unfall zugezogene Arzt findet noch keinerlei abnorme Veränderungen in der Lebergegend; 4 Wochen nach demselben konnte bereits ein mit dem unteren Leberrand in Zusammenhang stehender Tumor festgestellt werden. Bei der Probelaparotomie fanden sich sehr zahlreiche Verrwach'sungen des Tumors mit seiner Umgebung, die sehr wohl auf das stattgehabte Trauma bezogen werden konnten. Der Tumor hat sich dann sozusagen unter beständiger ärztlicher Kontrolle immer mehr vergrößert, die bald sich einstellende Kachexie nahm immer mehr zu, bis dann e in halbes Jahr nach dem Unfall der Tod eintrat. Autopsie und mikroskopische Untersuchung ergaben die Diagnose eines primären Spindelzellensarkoms der Leber.

Die traumatische Genese, der Kausalzusammenhang zwischen vorausgegangener Gewaltein. 
wirkung und der Entstehung der Neubildung schien so durchaus klargestellt zu sein. Unfallhergang und zeitlicherVerlaufentsprachend urch a us den Forderungender Unfallanerkennung, wie sie Thiem und Ka ufmann aufgestellt haben.

Zur Frage der traumatischen Genese der malignen Tumoren verhalten sich die Unfallchirurgen bekanntlich sehr reserviert. T h i e m betrachtet die Mitwirkung einer Verletzung bei der Entstehung eines Gewächses als ein außerordentlich seltenes Ereignis (höchstens 2 Proz. aller Krebse und 5 Proz. aller Sarkome). Noch zurückhaltender äußert sich $\mathrm{S}$ ter $\mathrm{n}$.

Die $\mathrm{klinischen} \mathrm{Statistiken} \mathrm{gelangen} \mathrm{allerdings} \mathrm{zu}$ wesent. lich höheren Zahlen. Es fanden:

Liebe (I.-D. Straßburg 1872-I881): 7,I4 Proz.

Rapok (I.-D. Straßburg 1880-1889): 33 "

Machol (I.-D. Straßburg I894-I899): 2,06"

Wolff (I.-D. Berlin 1874):

L e ugnitz (I.-D. Königsberg):

Deilmann (I.-D. Halle I903):

Kirchner (I.-D. München 1885 ):

Wild (I.-D. München I89I):

H e chinger (I.-D. München 1903): I8,3 " (nur Sarkome)

M e n ne berechnet aus verschiedenen Statistiken ein Mittel von 12,32 Proz. Die größtangelegte Statistik von Löwenthal ergibt 44,7 Proz.

Die von $\mathrm{Thiem}$ und $\mathrm{Ka}$ uf $\mathrm{mann}$ formulierten Bedingungen zur Annahme der traumatischen Entstehung maligner 'Tumoren:

I. Es muß ein Unfall in Gestalt einer Gewalteinwirkung - als bestimmtes zeitlich abgegrenztes Ereignis (K a u f $\mathrm{m}$ a $\mathrm{n} n$ ) - nachgewiesen sein.

2. Die Gewalteinwirkung muß eine einigermaßen erhebliche gewesen sein. Nötig sind dabei sinnfällige Einwirkungen, Schwellung, Blutunterlaufung, Schmerz, Schwäche (T h i e m).

3. Die Gewalteinwirkung muß das Organ oder die Körperstelle, wo sich später die Geschwulst entwickelt hat, auch getroffen haben.

4. Die zeitlichen Abhängigkeitsverhältnisse müssen mit den wissenschaftlichen Erfahrungen übereinstimmen. (Nach Th i e m nicht unter 3 Wochen.)

waren in unserem Falle erfüllt. - Ebenso die weitere Forderung $\mathrm{H}$ artmanns, daß der Zeitraum zwischen Unfall und deutlichem Auftreten der Geschwulst so groß sein müsse, daß während desselben die Geschwulst gemäß ihrer Wachstumstendenz sich entwickelt haben könnte, Für die Sarkome ist dabei die Angabe Theilhabers (zit. nach $\mathrm{K}$ a u f m a n n) wichtig, daß von 324 traumatischen Sarkomen 
$180=55$ Proz. unmittelbar im AnschluB an die Verletzung auftraten.

Th i em schlägt vor, als $\mathrm{k}$ ürzeste Frist zwischen Unfall und erster Manifestation für das Sarkom 3 Woch en anzunehmen. Wird also eine Geschwulst in noch kürzerer Zeit manifest, so muß sie schon vor dem Unfall bestanden haben.

Die Angaben der einzelnen Autoren über den zur Unfallsanerkennung nötigen Zeitraum zwischen Trauma und Sarkombildung schwanken überhaupt innerhalb weiter Grenzen.

Th i e m nimmt als längste Frist 2 Jahre an,

Deilmann als untere Grenze 3 Wochen, als obere 3 Jahre,

v. Bramann als untere Grenze 3 Wochen, als obere 2 Jahre (zit. nach $\mathrm{T}$ h i e $\mathrm{m}$ ).

Nach der Tabelle von Li e be (l.c.) sind bei 75 Sarkomfällen nach Trauma entstanden :

$\begin{array}{ll}\text { in I Monat und darunter: } & 34 \text { Sarkome } \\ \text { I Monat bis I Jahr: } & 27 \\ \text { mehr als I Jahr: } & \text { I4 ", }\end{array}$

Die größte Statistik von Lö wenthal enthält folgende Zahlen: Das Sarkom trat auf I Monat und darunter: in I35 Fällen

$\begin{array}{ll}\text { I Monat bis I Jahr: } & \text { in } 33 \text { " } \\ \text { mehr als I Jahr: } & \text { in } 22, "\end{array}$

Merkwürdig ist nach alledem eine von $\mathrm{T}$ hi e $\mathrm{m}$ zitierte Entscheidung des Reichsversicherungsamtes vom 22. II. I907, das ursächlichen Zusammenhang eines Riesenzellen-Sarkoms am untern Teil des Rückens nach einer Quetschung durch herabfallenden Stein von Kopfgröße vor 12 Jahren (1) annimmt.

Eine sinnfällige Einwirkung, entsprechend der zweiten Forderung Thiems, soweit sie Schwellung und Blutunterlaufung verlangt, war ja allerdings bei unserer Patientin a priori nicht vorhanden, bzw. im ärztlichen Befund nicht erwähnt. Thiem selber macht aber darauf aufmerksam, daB ein Schlag Tiefenwirkung haben kann, ohne daß die derbere Haut Zeichen einer Verletzung oder eines Blutergusses zu zeigen braucht. Man könne sich daher auch mit einer der angeführten sinnfälligen Erscheinungen (Schmerz, Schwindel) begnügen, und die waren in unserem Falle vorhanden. - Ebenso vor allem aus objektiven Veränderungen und subjektiven Beschwerden zusammengesetzte $Z$ wischenerscheinungen zwischen Unfall und Geschwulst (,Brückenerschein un . ge $n$ im Sinne Thiem s).

Vorsichtig gemacht durch die sehr zurückhaltenden Urteile von Thiem, Kaufmann, Stern, Hartmann, (Steinthal) schien uns bei der kritischen Durchsicht der Krankengeschichte nun aber doch die Körperkraft eines sehr grazil ge- 
bauten Jungen von 7 Jahren zu der von der Patientin geschilderten Wucht des Bürstenwurfes nicht recht passen zu wollen. Der Worte Th i e ms, ,jeder kleine Sto B, denen wir im Leben so oft ausgesetzt sind, kann kein Gewächs zur Folge haben", eingedenk, wurden nochmals nachträglich Erhebungen angestellt, die - wie eingangs erwähnt - zu der überraschenden Feststellung führten, daß die Patientin vor dem als direkte Ursache der Lebcrgeschwulstentwicklung angesprochenen Unfall sich nicht $\mathrm{meh} r$ recht wohl gefühlt, öfters über Schmerzen in der L ebergegend und über Magenstörungen, Appetitlosigkeit, schlechten Schlaf geklagt habe.-Weiter stellte sich heraus, da 3 die Patientin schon Ende Juli, a l s o s c h o n $6 \mathrm{Woch}$ en vor dem Unfall, dieser Beschwerden wegen einen Arzt aufgesucht hatte, der einen scheinbar unter dem Leberrand gelegenen klein en T u mor feststellte und denselben als Wan derniere ansprach. Von alledem hatte die Patientin bei der Anamnese kein Wort erwähnt. Es war dabei wohl weniger böse Absicht als die Ansicht der Patientin daran schuld, daß diese Dinge unwesentlich seien.

Es besteht nun allerdings ein gewisser Widerspruch in dem Ergebnis der beiden ärztlichen Befunde vor und nach dem Unfall. Während der eine Arzt schon Ende Juli einen kleinen Tumor in der nachher vom Unfall betroffenen Oberbauchgegend konstatierte, fand der 14 Tage nach demselben konsultierte Kollege keinerlei abnorme Veränderungen daselbst. Allein - abgesehen von hier nicht zu erörternden Imponderabilien der Untersuchungstechnik - schon die nachträglich als sicher vorhanden festgestellten subjektiven Beschwerden der Patientin vor dem Unfall müssen die Überzeugung aufdrängen, $d a B d$ er $T$ u mor $s c h$ on vor dem Unfall in seinen Anfängen bestanden habe. -

Eine lehrreiche Mahnung mehr, in Unfallfällen die Anamnese möglichst sorgfältig aufzufassen und nichts zu unterlassen, was zu einer objektiven Klarstellung beitragen kann. Gestützt nur auf klinische Anamnese und Befund mußte unser Fall geradezu als Schulbeispiel eines Sarkoms nach Trauma gelten; die Statistik nach Trauma entstandener Tumoren wäre um einen weiteren „sichern“ Fall bereichert gewesen. - In Wirklichkeit kann wohl 
ein begründeter $Z$ weifel nicht mehr bestehen, daß der Unfall höchstens eine Verschlimmerung eines schon vorher bestandenen Lebersarkoms herbeigeführt hat. Die durch den Schlag herbeigeführten sinnfälligen Einwirkungen (Ohnmacht, Schwindel und Schmerzen) lassen sich zusammen mit dem Sektionsbefund wohl zwanglos so erklären, daß das Trauma eine Bl ut ung in den Tumor bewirkte. - Ob nun der BluterguB erst Anlaß zu der bei der Autopsie festgestellten Zerfallscyste gegeben hat, oder der Blutaustritt in eine schon vorhandene Höhlung hinein erfolgt ist, kann für die Beurteilung des G r a de s der' Verschlimmerung nicht ganz irrelevant sein. - Die letztere Annahme müßte ja ein schon ziemlich weit vorgeschrittenes Wachstumsstadium zur Voraussetzung haben; eine erhebliche Verschlimmerung - etwa im Sinne einer zeitlichen Beschleunigung des tödlichen Ausgangs - käme wohl für ein rasch wachsendes Sarkom mit sowieso ganz kurzer Krankheitsdauer nicht mehr in Frage.

Anamnese und der beim Spitaleintritt erhobene Befund lassen es uns als sehr wahrscheinlich erscheinen, daß der durch den Unfall ohne Zweifel ursächlich bedingte Blutergu $B$ erst $A$ n l a $B$ zu einer Zerfallsbildung und damit zu rascherem Wachstum der Geschwulst gegeben hat.

Einen erheblichen erwerblichen Schaden im Sinne der Unfallgesetzgebung hat der Unfall zweifelsohne bedingt; bis dahin war die Patientin als Dienstmädchen voll arbeitsfähig, unmittelbar nachher dauernd arbeitsunfähig; der Zeitpunkt des Eintrittes der absoluten Erwerbsunfähigkeit wurde durch das Trauma erheblich vorgerückt. Es ist nach dem damals erhobenen Befund nicht wohl anzunehmen, daß die Sarkomentwicklung in der Leber bereits eine derart fortgeschrittene war, daß sie auch ohne Unfall in kurzer Zeit zur vollständigen Erwerbsunfähigkeit und zum Tode geführt hätte. -

In der reichhaltigen Kasuistik der Sarkomfälle nach Trauma bei Thiem und $\mathrm{Kaufmann}$ ist kein Fall von Lebersarkom erwähnt. In der ganzen Literatur - soweit sie mir zugänglich war - habe ich nur 4 Fälle erwähnt gefunden, in denen ein ursächlicher Zusammenhang zwischen Trauma und Entstehung eines primären Lebersarkoms in Frage kommt, behauptet oder ange- 
nommen wird; die Krankengeschichten seien am Schlusse der Arbeit kurz zusammengestellt.

Wag ner berichtet über einen 5 jährigen Jungen, der 8 Tage (!) bevor der Arzt eine Lebergeschwulst feststellte - cinen Schlag auf den Bauch erhielt. Vorher sei der Knabe nie erheblich unwohl gewesen.

Bei Arnold handelt es sich um einen 15 jährigen Knaben mit primärem Lebersarkom, der -8 Wochen bevor er bettlägerig wurde - in die rechte Seite des Leibes getreten wurde, worauf er mehrere 'Гage starke Schmerzen beim Atmen hatte.

$B$ är ensprung erwähnt den Fall eines Unteroffiziers, der - ca. $1 \frac{1}{2}-2$ Monate, bevor klinisch eine Lebergeschwulst konstatiert wurde - einen Schlag in die Magengegend erhielt.

Bei der Beobachtung, die $\mathrm{S} c \mathrm{~h}$ e i d e $\mathrm{m}$ a $\mathrm{n} \mathrm{d}$ el in seiner Inaug.Diss. verwertete, ist nach der publizierten Krankengeschichte wiederum die lehrreiche Tatsache festzustellen, daß die klinische Anamnese (siehe Krankengeschichte) ebenfalls nachträglich dahin ergänzt wurde, daß schon vor de m - mitder Sarkombild ting als im $Z$ us ammenhang stehend angenommenen - Unfall der betreffende Patient sich nicht wohl fühltc und über Schmerzen in der rechten Seite geklagt hatte.

Die Fälle Wagner und Scheidemandel (l. c.) fallen ohne weitercs außer Betracht. Es ist klar, daB - nach den früher angefuihrten und allgemein anerkannten Forderungen von $T \mathrm{~h}$ i e $\mathrm{m}$ und Kaufmann, namentlich was die zeitlichen Bez i eh ungen anbetrifft, ein Lebersarkom nicht - wic im Falle W a g ner - als durch Lnfall bedingt anzusehen ist, wenn schion 8 Tage nach demselben ein Lebertumor konstatiert wird. 'T he il h a ber (l. c.) allerdings vertritt - wie schon früher erwähnt eine gegenteilige Auffassung.

Die Angaben Bärensprungs sind leider zu unvollständig, als daß sie ein einigermaßen sicheres Urteil erlauben würden.

Im Falle Arnolds dagegen - die Richtigkeit und Vollständigkeit der anamnestischen Angaben vorausgesetzt - ist ein Zusammenhang zwischen Unfall und Sarkombildung in der Leber wenigstens nicht von vornherein von der Hand zu weisen. Einige 
Wochen erst nach dem Unfall wurde bei dem bis dahin angeblich ganz gesunden Handlanger ein hühnereigroßer Lebertumor konstatiert. Die von Thiem aufgestellte untere Grenze wäre demnach nicht überschiritten.

Angesichts von Erfahrungen, wie sie Scheidemandel und wir gemacht haben, des Standpunktes, den Autoritäten wie 'Thiem, Kaufmann, Stern, Steinthal (Hartmann), $\mathrm{L} u \mathrm{~b}$ a rsch einnehmen, ist Vorsicht auch hier geboten, wenn auch die Ansicht Steinthals, ,daß eine traumatische Entstehung von Sarkomen zurzeit noch der wissenschaftlichen Unterlage entbehrt", wohl nicht allgemein geteilt werden dürfte. -

Dic Krankengeschichten von Wagner, Arnold, Bärensprung und Scheidemand el mögen hier auszugsweise angeführt sein. -

1. Wagner in Birch-Hirschfelds Leberkrankheiten in Gerhardts Handbuch für Kinderkrankheiten I865, IV, 2, S. 80.

5jähriger, schwächlich gebauter, schlecht ernährter Knabe von blasser Gesichtsfarbe. Acht Tage, bevor der Arat eine Lebergeschwulst feststellte, erhielt der Knabe einen Schlag auf den Bauch. Vorher sei Pat. nie erheblich unwohl gewesen. Beginn der Krankheit mit Erbrechen und Durchfall, Bauch etwas aufgetrieben, Hautvenen erweitert. Rechts vom Rippenkorbrand bis unterhalb des Nabels fand sich eine höckerige Geschwulst, der vergrößerten Leber entsprechend. In der folgenden Zeit rasche Zunahme der Geschwulst. Pat. magerte $a b$; es trat Schmerzhaftigkeit des ganzen Bauches und Atemnot ein. Urin enthiclt Eiweiß, Ikterus und Ascites fehlte. Zwei Monate nach dem Auftreten der ersten Krankheitserscheinungen trat der Tod ein. Klinische Diagnose: Leberkrebs. Bei der Sektion fand man die Leber stark vergröBert, die vordere Hälfte der Bauchhöhle einnehmend. Auf der Oberfläche und dem Durchschnitt zahlreiche grauweiße Knoten, besonders seitlich längs der Pfortaderäste. Auch in den Venen zahlreiche Knötchen. - Mesenterium, Bauchfell und Darmwand waren von Geschwulstmassen infiltriert. Die mikroskopische Untersuchung ergab als Hauptbestandteil Zellen von der Beschaffenheit der farblosen Blutkörperchen in den Lücken eines zarten Netzwerkes, mit einem ausgebildeten Kapillargefäßsystem. Wahrscheinlich handelte es sich um Lympho-Sarkom. -

2. Arnold: Zwei Fälle von primärem Angio-Sarkom der Leber. ( $Z$ i e glers Beitr. z. patholog. Anatom. usw. Bd. 8, S. 123).

I 5 jähriger Knabe, von gesunden Eltern stammend, früher stets gesund, erkrankte mit starken, von Tag zu Tag sich steigernden Schmerzen in der Oberbauchgegend. Acht Wochen bevor er bett- 
lägerig wurde, sei er in die rechte Seite des Leibes getreten worden, worauf er mehrere Tage starke Schmerzen beim Atmen hatte. Er arbeitete weiter, bemerkte aber bald eine auf Druck und spontan schmerzhafte Schwellung rechts vom Nabel unterhalb des Rippenkorbrandes, die rasch zunahm und ihn zwang, im Bett liegen $z \mathrm{u}$ bleiben. Etwa 3 Wochen nach dem Auftreten der ersten Krankheitserscheinungen. fühlte der behandelnde Arzt einen hühnereigroßen, sowohl spontan als auch bei Druck schmerzhaften Tumor in der Regio hypochondrica.

Klinischer Befund: Schwächlich gebauter, blasser Knabe. Haut nicht ikterisch. In der rechten Seite des Leibes ein bis nahe an die Crista ilei reichender Tumor, der sich bei der Respiration wenig verschiebt. In der Bauchhaut zahlreiche geschlängelte Venen. Leberdämpfung reicht von der 5. Rippe bis zur Crista ilei. Keine Sehstörungen; Augenspiegeluntersuchung ergibt auf beiden Seiten normalen Befund. Einige, bei der Probepunktion entnommene Partikelchen zeigen mikroskopisch die typische Struktur des AngioSarkoms. Bei der später vorgenommenen Probelaparotomie fand man dic Leber, soweit übersehbar, mit unzähligen markigen, kirschkerngroßen Knoten durchsetzt. Exitus 2 Tage post operat. -

Bei der Sektion reicht die Leber ca. $4 \mathrm{~cm}$ unter den Nabel und steht fast in gleicher Höhe mit der Spina ant. sup. ilei. Gewicht der Leber $3125 \mathrm{~g}$. Auf dem Durchschnitt zeigt sich der rechte Leberlappen von einer Unzahl kleinster bis nußgroßer, graurötlicher Tumoren durchsetzt. Reste von Lebergewebe sind nur noch in den untern Abschnitten vorhanden. - Die interacinösen Bindegewebszüge sind hier verbreitert. Im linken Leberlappen keine Knoten. Pfortader bis zur Vena lienalis mit schmieriger graurötlicher Masse angefüllt. - Die übrigen Organe zeigen keine bemerkenswerten Veränderungen; in keinem derselben finden sich Geschwülste.

Mikroskopischer Befund: Linker Leberlappen von starken Bindegewebszügen durchsetzt; Leberzellen enthalten teilweise Fett. Gefäße und Gallengänge in großer Zahl vorhanden. Ähnlicher Befund im rechten Leberlappen; hier finden sich zwischen den Leberzellen Anhäufungen von Rundzellen. Die Geschwulstknoten selbst zeigen den typischen Bau eines alveolären Angiosarkoms. Die Entwicklung des Geschwulstgewebes schien im Anschluß an die GefäBe der interacinösen Bindegewebszüge in der Art zu erfolgen, daß in den Gefäßscheiden Zellen sich anhäuften, die durch Vermehrung und Wachstum zu Knollen und Haufen yon Sarkomzellen sich umwandeln.

3. Bärensprung (Deutsche militärärztliche Zeitschrift 189I, zit. nach L.̈̈wenthal).

Ein Unteroffizier erhielt Nov. 1883 einen Schlag in die Magengegend. Kurze Zeit darauf, nachdem seit dem Schlage Appetitlosigkeit und Magenschmerzen bestanden hatten, wird in der Magengrube 
eine anscheinend der Haut angehörende Infiltration von Zehnpfennigstückgröße konstatiert. Am 9. I. 1884 fühlt man an der Stelle der Infiltration eine flache, harte, bei der Atmung auf- und absteigende Geschwulst. Stetige Vergrößerung derselben und der ganzen Leber, dabei starke Schmerzen in der rechten Seite und Schlaflosigkeit, dazu starker Ascites. - Am 17. V. 1884 Tod an Entkräftung. -

Sektionsbefund: Enorme Vergrößerung der brettharten Leber, besonders des linken Leberlappens (40:29: I8). Durchsetzung des braunroten Gewebes mit zahllosen kleinen und größeren, weißen, vorspringenden, harten Knoten. Keine Metastasen.

Mikroskopischer Befund: Rund- und Spindelzellensarkom.

4. Scheidemandel: Ein Fall von primärem Sarkom der Leber. (Inaug.-Diss. Erlangen I903).

8 jähriger Knabe fiel beim Spielen von einem Bretterhaufen hinunter, worauf Patient starke Schmerzen in der Seite hatte. Von da an war der Knabe in abwechselnder Stärke krank mit teilweise sehr hohem Fieber, ohne daß ein eigentlicher Befund erhoben werden konnte. Bei genauer Nachfrage geben die Eltern des Patienten noch an, daß schon etwa I4 Tage vor dem Fall das Kind nicht recht wohl gewesen sei, und über Schmerzen in der rechten Seite geklagt habe, dabei aber noch die Schule besuchte. - Drei Wochen nach dem Unfall wird folgender Befund erhoben:

Stark abgemagertes, anämisches Kind. - Über der rechten Bauchseite sind die Venen bedeutend stärker gefüllt als links. Das rechte Epigastrium ist etwas aufgetrieben, die rechte Seite auf Druck sehr empfindlich. Leberdämpfung vergrößert. Temperatur 39,5 Grad. Bei einer Punktion in der vorderen Axillarlinie des 9. Interkostalraumes füllt sich die Spritze leicht mit blutig-seröser Flüssigkeit; es werden darin viel Eiweiß, viel Inosit, kein Harnstoff, kein bernsteinsaurer Kalk oder Na gefunden. Im Sediment reichlich Erythround Leukocyten, keine Haken.

In der nächsten Zeit wurde die Auftreibung der rechten Seite sehr beträchtlich, und ragte nun weit über den Rippenbogen herab. Da man ein sekundär infiziertes Hämatom vermutete, wird eine Woche nach dem Eintritt in ärztiiche Behandlung operiert: Schnitt parallel dem Rippenbogen. Leberoberfläche mit den Bauchdecken verwachsen; bei der Inzision wird eine Cyste eröffnet, ohne die freie Bauchhöhle zu berühren. Es entleert sich ungefähr I Liter blutig-seröser Flüssigkeit. Mit dem eingeführten Finger kommt man in eine kindskopfgroße Höhle mit weichen fetzigen Wandungen. Tamponade. Unter zunehmender Schwäche Exitus I I Tage später. -

Sektion: Beide Lungen durch den Zwerchfellhochstand komprimiert. Im rechten Unterlappen ein ziemlich großer hämorrhagischer Infarkt. Im Mediastinum kleinere metastatisch infiltrierte Drüsen- 
pakete, desgleichen solche der Zwerchfellkuppe aufgelagert, eine Reihe kleiner flacher Geschwulstknötchen. Die stark vergrößerte Leber ist mit dem $Z_{w}$ erchfell und der vordern Bauchwand fest verwachsen. Die Vergrößerung der Leber betrifft hauptsächlich den rechten Lappen. Gewicht 2250 g. Rechter Leberlappen mißt I 5:20:14. Gallenblase intakt, enthält etwas dickflüssige Galle. Auf mehreren durch die Leber gelegten Durchschnitten zeigt sich im rechten Leberlappen ein fast kreisrunder, den ganzen Lappen in zylindrischer Form durchziehender Tumor mit einem Durchmesser von $8 \mathrm{~cm}$. An der vordern und lateralen Seite reicht derselbe bis an die Kapsel, während er sich nach links in eine innen fetzige Cyste fortsetzt, die den rechten und linken Lappen verbindet. Die Hauptmasse des Tumors besteht aus dunkelrotem, weichem Gewebe, das gegen die Kapsel zu eine markigweiße Farbe annimmt. Gegen das Lebergewebe $z \mathfrak{u}$ ist der Tumor durch eine ganz zarte, bindegewebige Schicht abgegrenzt, ebenso ist der Tumor in seinem Innern von bindegewebigen zarten Cysten durchzogen, so daß er ein alveoläres Aussehen bekommt.

Mikroskopische Untersuchung: Die Geschwulstzellen sind wenig größer wie Lymphocyten und haben einen kleinen Zellleib mit runden, teils ovalen Kernen. Zwischen den Zellen sind nur geringe Spuren von Zwischensubstanz. - Im Bereiche der Geschwulst finden sich $a b$ und zu Züge eines lockeren Bindegewebes, in dem kleine, mit Erythrocyten angefüllte Gefäße verlaufen. Sehr gefäßreich sind insbesondere die in den Tumormassen zerstreut liegenden vollständig nekrotischen Partien, in denen nur noch Andeutungen von Zellbestandteilen bestehen. Das Lebergewebe zeigt sich konzentrisch verdrängt und zum großen Teil atrophisch; einige Leberbalken treten durch starke Erweiterung der Kapillaren auseinander. Auf Schnitten, die Teile des $Z$ werchfelles mitgenommen haben, sieht man mitunter, wie sich Geschwulstzellenreihen zwischen die quergestreiften unveränderten Fasern der Zwerchfellmuskulatur hineinschieben.

Die mikroskopisch untersuchten Mediastinal-Lymphdrüsen ergaben ebenfalls die Diagnose: Rundzellensarkom. -

Was die unfallrechtliche Seite anbetrifft, so finden in concreto die speziellen Haftpflichtgesetze bei dem Fall des an primärem Lebersarkom verstorbenen $D$ ienstmädchens $\mathrm{k}$ cinc Anwendung. ${ }^{1}$ )

Angesichts des ärztlichen Befundes haben die Hinterbliebenen

I) Ich verdanke diese Auskünfte einer freundlichen Mitteilung von Herrn Dr. jur. K. Saas in Winterthur, dem ich auch an dieser Stelle meinen besten Dank aussprechen möchte. 
des Dienstmädchens weder nach deutschem noch nach schweizerischem Rechte $\Lambda$ ussicht, Ersatzansprüche gegen den Knaben oder seinen Vater mit Erfolg geltend machen zu können. Die Voraussetzungen der in Betracht fallenden $\$ \S 823,828,829$ und 832 des BGB. fehlen; so vor allen Dingen der haftungsbegründende Kausalzusammenhang. Nach der Rechtsprechung des Reichsgerichts liegt Verursachung vor, ,wenn ohne den Umstand der Schaden überhaupt nicht oder doch nicht so wie geschehen eingetreten wäre und der Umstand die Möglichkeit des Eintrittes eines Erfolges von der Art des eingetretenen erheblich erhöht hat". Dieser Kausalzusammenhang muß mindestens in einem ,jeden vernünftigen $Z$ weifel ausschließenden Grade von Wahrscheinlichkeit" dargetan werden, z. B. durch Darlegung eines Umstandes, ,auf den nach dem gewöhnlichen Laufe der Dinge der eingetretene Schaden zurückzuführen ist". (Wolf Handkommentar zum BGB., I909, S. 59.) Darnach ist ohne weiteres klar, daß der Wurf mit der Bürste als haftungsbegründende Ursache weder für den Tod noch für die Verkürzung der Lebensdauer des Mädchens bezeichnet werden darf; denn laut dem ärztlichen Befund bestand die Todesursache schon vor dem Wurf und läßt sich bezweifeln, ob der Wurf den Tod wirklich beschleu. nigt hat.

Aber auch wenn ein Kausalzusammenhang im Sinne der Rechtsprechung des Reichsgerichtes vorläge, so würde doch die weitere Voraussetzung des Verschuldens mangeln; denn der Knabe lätte den eingetretenen Erfolg natürlich weder gewollt noch ihn als einen rein zufälligen voraussehen können; d. l. er hätte weder vorsätzlich noch fahrlässig gehandelt, \$823; er wäre zudem schon deswegen nicht verantwortlich, weil er als Siebenjähriger unmöglich dic Einsicht besitzen konnte, der Wurf nit der Bürste sei so gefährlich, daß er zu einem Sarkom führen könne.

Mangels Kausalzusammenhanges ist aber nicht nur die Ersatzpflicht aus $\S 823$ ausgeschlossen, sondern auch die sogenannte Billigkeitshaftung aus $\$ 829$; denn $\S 829$ sicht zwar vom Frfordernis des Verschuldens ab, enthält aber wie $\$ 823$ die Bedingung, daß der Schaden „verursacht" worden sei.

Aus dem gleichen Grunde müßten Ersatzansprüche gegen den Vater des Knaben als aufsichtspflichtigen Inhaber der elter- 
lichen Gewalt abgewiesen werden; denn die Ersatzpflicht gemäß $\$ 832$ beruht wiederum auf der hier fehlenden Voraussetzung des Kausalzusammenhanges. Zudem entfällt die Ersatzpflicht, wenn der Aufsichtspflicht über den Minderjährigen Genüge getan war; das Gegenteil zu beweisen, dürfte den Klägern schwer fallen, da dic Aufsicht über den Knaben von den Eltern für den Fall ihrer Abwesenheit wohl gerade dem Mädchen überbunden war.

Nach Art. 95 des Einführungsgesetzes zum BGB. bleiben allerdings dic landesgesetzlichen Vorschriften des Gesinderechts unberührt. Es darf aber angenommen werden, daß einschlägige Bestimmungen des Gesindelandrechts mit Sicherheit den Kausalzusammenhang und wahrscheinlich auch das Verschulden als Voraussetzungen eines Haftbarkeitsanspruches enthalten. -

Das s chweizeris che R c cht führt zum gleichen Ergebnis. In Betracht fallen Art. $4 \mathrm{I}$ ff. des Obligationenrechtes in Verbindung mit Art. 19 Abs. 3 und Art. 333 des Zivilgesetzbuches. Mangels Kausalzusammenhanges und Verschuldens haften weder der Knabe noch sein Vater (als aufsichtspflichtiges Familienhaupt) für den Tod oder für die Verkürzung der Lebensdauer des Mädchens. -

Für die Überlassung des Falles, für das freundliche Interesse und die Unterstützung, die sie meiner Arbeit entgegenbrachten, bin ich meinem hochverehrten Chef, Herrn Direktor Dr. Stier 1 in und meinem verehrten ehemaligen Lehrer, Herrn Prof. Dr. O. Bus se, zu großem Danke verpflichtet.

\section{Literaturverzeichnis.}

1. Arnold, Zwei Fälle von primärem Angiosarkom der Leber. Zieglers Beitr. z. patholog. Anatom. I89o, Bd. 8, S. 123.

2. Bärcnsprung. Deutsche militärärztl. Zeitschr. I89I, zit. nach Löwenthal.

3. B a y er, Zwei Fälle von primärem Sarkom der Gallenblase. Zieglers Beitr. z. patholog. Anatom. usw. 1904, Bd. 46, S. 429.

4. Bertelli, Contributo allo studio dei sarcomi primitivi del fegato. Policlinico, Sez. chir. 1908, XV, 6 u. 7; Ref. Zentralbl. f. Chir. 1908, S. 1323 .

5. Bramwell, Enormous prim. sarcoma of the liver etc. Edinb. med. Journ. I 896 , zit. nach Thöle. 
6. Chessin, Zur Frage der Verwendbarkeit der Fascia lata bei Leberresektionen. Zentralbl. f. Chir. I913, S. II73.

7. Cignozzi, Das elastische System in Lebercarcinomen. Clin. chirurg. I910, Nr. 2. Ref. Zentralbl. f. Chirurg. I9ro, S. 897.

8. Cornil et Ranvier. Histologie patholog. 1884, Bd. 2, S. 459, zit. nach Orth.

9. Deilmann, Über den Zusammenhang von Sarkom und Trauma. Inaug.-Diss. Halle 1903.

10. Garbarini, I tumori maligni del fegato e delle vie biliari etc. Ref. Zentralbl. f. Chir. I907, S. I382.

II. $\mathrm{H}$ a a s $\mathrm{l}$ e r, Bericht über den II. internat. Chirurgenkongreß. Zentralbl. f. Chir. 1907, S. I341.

I2. Hartmann, Beiträge zur Frage des Sarkoms nach Trauma. Bruns Beitr. z. klin. Chir., Bd. 88, S. 572.

13. $\mathrm{H} \mathrm{e} \mathrm{ch} \mathrm{ing} \mathrm{er} \mathrm{,} \mathrm{Über} \mathrm{traumatische} \mathrm{Entstehung} \mathrm{des} \mathrm{Sarkoms.} \mathrm{Inaug.-Diss.}$ München 1903.

I4. Hilse, Die freie Fettransplantation bei Blutungen der parenchymatösen Bauchorgane. Zentralbl. f. Chir. 1913, S. I849.

I5. Hot es, Ein Fall von primärem Sarkom der Gallenblase. Inaug.-Diss. Leipzig 19 Io.

I6. Kahlden, v., Über das primäre Sarkom der Leber. Zieglers Beitr. z. patholog. Anatom. I 897, Bd. 21, S. 264.

17. Ka u mann, Handbuch der Unfallmedizin 1915, Bd. 2, Unfallerkrankungen.

18. Kirschner, Über freie Sehnen- und Faszientransplantation. Bruns' Beitr. z. klin. Chir., Bd. 65, S. 472.

19. Knott, Primary sarkoma of the liver. Surg.-Gynaecol. and Obstetr. Vol. VII, Nr. 3; Ref. Zentralbl. f. Chir. 1908, S. I 542.

20. Kornew \& Scha a ck, Ein neues Verfahren für ausgedehnte Leberresektionen mit Anwendung der freien Fascientransplantation. Zentralbl. f. Chir. I913, S. 949.

2r. Landsteiner, Über das Sarkom der Gallenblase. Wiener klin. Wochenschr. 1904, S. I63.

22. LöwenthaI, Über die traumatische Entstehung der Geschwïlste. Langenb. Archiv f. klin. Chir., Bd. 49, S. I und 267.

23. Machol, Die Entstehung von Geschwülsten im Anschluß an Verletzungen. Inaug.-Diss. Straßburg 1900.

24. Marx, Über das primäre Sarkom der Leber. Zentralbl. f. allgem. Patholog. und patholog. Anatom. 1904, Bd. 15, S. 433.

25. Mazzoni, Amputatione del lobo sinistro del fegato per tumore. Bull. della Acad. med. di Roma I904, Bd. 30. Ref. Zentralbl. f. Chir. 1906, S. 96 .

26. M enne, Die Bedeutung des Traumas für das Entstehen und Wachstum der Geschwülste usw. Deutsche Zeitschr. f. Chir., Bd. 8I, S. 374.

27. $\mathrm{M}$ üller, Bericht über den 26. Chirurgenkongreß 1897, Bd. I, S. II8. Deutsche Zeitschrift f. Chirurgie. I 38 . Bd. 
34 DuBs, Über ein angeblich nach Trauma entst., primäres Sarkom der Leber.

28. N e gri, L'emostasi nella resezione del fegato. Clin. Chirurg. I9I I, Nr. I I. Ref. Zentralbl. f. Chir. I9I2, S. 507.

29. Opokin \& Schamoff, Zur Frage der blutstillenden Wirkung von Muskelgewebe bei Leberverletzungen. Zentralbl. f. Chir. 1913, S. 1217.

30. Orth, M., Über primäres Lebersarkom. Inaug.-Diss. Straßburg 1885 .

31. Picone, La chirurgia epatica dal punto di vista emostatico. Clin. Chir. 1914, XXII, 9. Ref. Zentralbl. f. Chir. 1915, S. 537.

32. Rolleston \& Salisbury, Primary sarkoma arising in a cirrhotic liver. Journ. of Pathol. and Bakteriol. XV, I9Io, S. 247. Ref. Schmidts Jahrb. 3I I, S. 149.

33. Scheidemandel, Ein Fall von primärem Sarkom der Leber. Inaug.-Diss. Erlangen 1903.

34. Schönlank, Ein Beitrag zur Kasuistik des primären Sarkoms der Gallenblase. Frankfurter Zeitschr. f. Pathol,, Bd. 15, H. 2.

35. Stern, Die traumatische Entstehung innerer Krankheiten.

36. Th i e $\mathrm{m}$. Handbuch der Unfallerkrankungen I909. Bd. I.

37. Thöle. Chirurgie der Lebergeschwülste in Neue Deutsche Chirurgie, I9I3, Bd. 7 .

38. W a g n er. Birch.Hirschfelds Leberkrankheiten in Gerhardts Handbuch f. Kinderkrankheiten I 865 , zit. nach Scheidemandel. IV, Bd. 2, S. 80.

39. W e nde l, Beiträge zur Chirurgie der Leber. Langenb. Archiv f. klin. Chir. I9I I, Bd. 95, H. 4. 